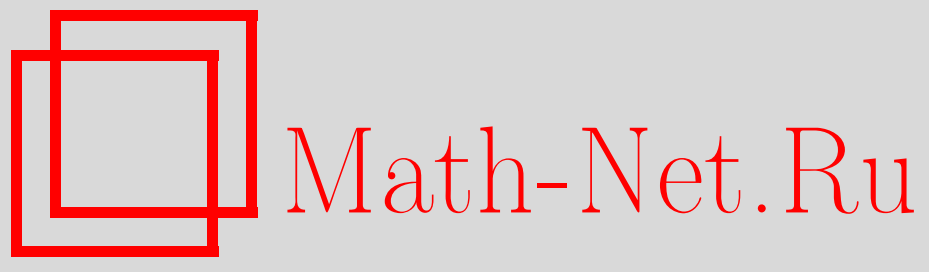

В. В. Голышев, О канонической полосе, УМН, 2009, том 64, выпуск 1, 139-140

DOI: https://doi.org/10.4213/rm9276

Использование Общероссийского математического портала Math-Net.Ru подразумевает, что вы прочитали и согласны с пользовательским соглашением http://www . mathnet.ru/rus/agreement

Параметры загрузки:

IP: 34.229 .108 .108

26 апреля 2023 г., $17: 05: 24$

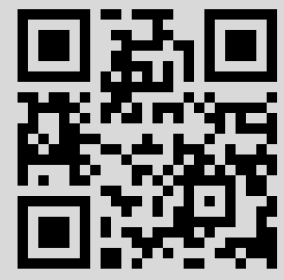




\section{О канонической полосе}

\section{В. В. Голышев}

Мы показываем, что из гипотезы о канонической полосе для многообразия Фано следует, что нули многочлена Гильберта сечения общего типа или типа Калаби-Яу в нем расположены на вертикальной прямой. В частности, на вертикальной прямой лежат нули многочлена Гильберта сечения общего типа или типа Калаби-Яу в грассманиане.

1. Каноническая полоса и гипотеза о канонической прямой. Пусть $H(z)-$ многочлен Гильберта многообразия Фано или общего типа $X$, т. е. $H(n)=H_{-K_{X}}(n)=$ $\chi(n(-K))$ для целых $n$.

(CS) Скажем, что $X$ удовлетворяет гипотезе о канонической полосе, если все корни $z_{i}$ многочлена $H(z)$ лежат в канонической полосе $-1<\operatorname{Re} z<0$.

(NCS) Скажем, что $X$ удовлетворяет гипотезе о суженной канонической полосе, если все корни $z_{i}$ многочлена $H(z)$ лежат в суженной канонической полосе

$$
-1+\frac{1}{\operatorname{dim} X+1} \leqslant \operatorname{Re} z \leqslant-\frac{1}{\operatorname{dim} X+1} .
$$

(CL) Скажем, что $X$ удовлетворяет гипотезе о канонической прямой, если все корни $z_{i}$ лежат на вертикальной прямой $\operatorname{Re} z=-1 / 2$.

Ясно, что $(\mathrm{CL}) \Longrightarrow(\mathrm{NCS}) \Longrightarrow(\mathrm{CS})$.

2. Вложенные многообразия Калаби-Яу. Для многообразия Калаби-Яу $X$ многочлен Гильберта относительно антиканонического класса не интересен. Поэтому мы скажем, что многообразие Калаби-Яу $X$, вложенное в многообразие Фано $F$ как антиканоническое сечение, удовлетворяет гипотезе о канонической прямой, если корни многочлена Гильберта относительно ограничения класса $-K_{F}$ на $X$ чисто мнимы.

Можно показать, что гипотеза (NCS) выполняется для многообразий Фано и минимальных многообразий общего типа в размерностях 1,2 и 3.

3. Грассманианы. Проективные пространства $\mathbb{P}^{n}$ удовлетворяют гипотезе о суженной канонической полосе: нули суть $-i /(n+1), i=1, \ldots, n$. Как показал $\Phi$. Хирцебрух [1], это обобщается на грассманианы. Пусть $G(k, N)$ - грассманиан $k$-плоскостей в $N$-мерном пространстве. Предположим, что $N \geqslant 2 k$. Пусть $\varphi(x)-$ кусочно линейная функция вещественного аргумента $x$, заданная равенством $\varphi(x)=$ $\min (k,-x, x+N)$. Тогда

$$
H(z)=c \prod_{i=1}^{N-1}\left(z+\frac{i}{N}\right)^{\varphi(-i)}
$$

4. Многообразия общего типа могут не удовлетворять (NCS). Ситуация, по-видимому, более специфична в случае вложенных многообразий.

Теорема. Пусть $F$ - многообразие Фано, удовлетворяющее гипотезе о канонической полосе, и пусть $X$ - его гладкое сечение общего типа (соответственно типа Калаби-Яу) в линейной системе $-n K_{X}, n>1$ (соответственно $\left.n=1\right)$. Тогда (CL) выполняется для вложенного многообразия $X$.

ДоказАтельство. Пусть $H_{F}(z)$ - многочлен Гильберта многообразия $F$. Обозначим через $H_{r}(z)$ многочлен Гильберта $X$ относительно ограничения $-K_{F}$. Для многообразия общего типа $X$ формула присоединения говорит, что антиканонический 
класс $X$ кратен ограничению антиканонического класса $F$, так что можно доказывать утверждение о вертикальной прямой для $H_{r}$. Имеем

$$
H_{r}(z)=H_{F}(z)-H_{F}(z-n) .
$$

Для доказательства того, что нули $H_{r}(z)$ лежат на прямой $\operatorname{Re} z=(n-1) / 2$, используется

Лемма. Пусть многочлен $H(z) \in \mathbb{R}[z]$ таков, что

i) $H(-1-z)= \pm H(z)$,

ii) все его корни $z_{i}$ находятся в открытой левой полуплоскости.

Тогда для любого вещественного $s \geqslant 1$ и для любого корня $\zeta_{j}$ многочлена $H(z)-$ $H(z-s)$ имеем $\operatorname{Re} z=(s-1) / 2$.

ДокАзАтЕЛЬСтво. Предположим, что найдется корень $\zeta=\zeta_{j}$ многочлена $H_{r}(z)$ такой, что $\operatorname{Re} \zeta<(s-1) / 2$. По предположению,

$$
\prod\left(\zeta-z_{i}\right)=\prod\left(\zeta-z_{i}-s\right)
$$

Пусть $\mu_{s}$ - отображение, переводящее $z$ в $(s-1)-\bar{z}$. Оно устанавливает взаимно однозначное соответствие между сомножителями в левой и правой частях:

$$
\prod\left(\zeta-z_{i}\right)=\prod\left(\zeta-\mu_{s}\left(z_{i}\right)\right) .
$$

Однако для любого $i$ выполняется неравенство

$$
\left|\zeta-z_{i}\right|<\left|\zeta-\mu_{s}\left(z_{i}\right)\right|
$$

так как $\zeta$ находится слева от оси симметрии $\mu$.

Так же разбирается случай $\operatorname{Re} \zeta(s-1) / 2$. Это противоречие доказывает лемму и теорему.

СлЕДСтвиЕ. Сечение линейной системы $-m K_{F}, m>0$, на грассманиане $F$ удовлетворяет гипотезе о канонической прямой.

5. Вопросы. А. Существуют ли многообразия Фано (соответственно многообразия общего типа), не удовлетворяющие гипотезе о канонической полосе? Как охарактеризовать многообразия, удовлетворяющие (CS), но не (NCS)?

Б. Исследовать нули многочленов Эрхарта [2] для политопов типа Фано в сравнении с нулями многочленов Эрхарта политопов, не являющихся политопами типа Фано. Как их охарактеризовать?

В. Начиная с размерности 4 , классический критерий Рауса-Гурвица доставляет набор полиномов от чисел Чженя многообразия, чья положительность/неотрицательность влечет (CS) или (NCS). Достаточно ли одной теоремы Яу, чтобы доказывать неравенства этого типа, или мы нуждаемся в целой иерархии ее усилений?

Я признателен Д. Загиру, рассказавшему мне о работе Ф. Родригеса-Виллегаса [3], которая мотивировала написание этой заметки. Я благодарю Ф. Хирцебруха за обсуждение, а также И. А. Чельцова и К. А. Шрамова за их советы.

\section{Список литературы}

[1] F. Hirzebruch, Semin. Analytic Functions, 2 (1958), 92-104. [2] M. Beck, J. A. De Loera, M. Develin, J. Pfeifle, R.P. Stanley, Integer points in polyhedra - geometry, number theory, algebra, optimization, Contemp. Math., 374, Amer. Math. Soc., Providence, RI, 2005, 15-36. [3] F. Rodriguez-Villegas, Proc. Amer. Math. Soc., 130:8 (2002), 2251-2254.

В. В. Голышев (V. V. Golyshev)

Математический институт им. В. А. Стеклова РАН

E-mail: golyshev@mccme.ru
Представлено В. М. Бухштабером Принято редколлегией 12.12 .2008 